\title{
Study on the Mechanical Instability of MICE Coupling Magnets
}

\author{
L. Wang, H. Pan, X. L. Guo, H. Wu, S. X. Zheng, M. A. Green Member, IEEE
}

\begin{abstract}
The superconducting coupling solenoid magnet is one of the key equipment in the Muon Ionization Cooling Experiment (MICE). The coil has an inner radius of $750 \mathrm{~mm}$, length of $281 \mathrm{~mm}$ and thickness of $104 \mathrm{~mm}$ at room temperature. The peak induction in the coil is about $7.3 \mathrm{~T}$ with a full current of 210 A. The mechanical disturbances which might cause the instability of the impregnated superconducting magnet involve the frictional motion between conductors and the cracking of impregnated materials. In this paper, the mechanical instability of the superconducting coupling magnet was studied. This paper presents the numerical calculation results of the minimum quench energy (MQE) of the coupling magnet, as well as the dissipated strain energy in the stress concentration region when the epoxy cracks and the frictional energy caused by "stick-slip" of the conductor based on the bending theory of beam happens. Slip planes are used in the coupling coil and the frictional energy due to "slow slip" at the interface of the slip planes was also investigated. The dissipated energy was compared with MQE, and the results show that the cracking of epoxy resin in the region of shear stress concentration is the main factor for premature quench of the coil.
\end{abstract}

Index Terms-Friction, minimum quench energy, strain energy, superconducting magnets

\section{INTRODUCTION}

$\mathrm{T}^{\mathrm{s}}$ HE superconducting coupling solenoid is to be used for the Muon Ionization Cooling Experiment (MICE) which will be a demonstration of muon cooling for a future neutrino factory [1]. The coupling coil made from copper matrix NbTi conductors is the largest of three types of magnets in the MICE cooling channel both in terms of $1.5 \mathrm{~m}$ inner diameter and about $7.3 \mathrm{~T}$ peak magnetic field at full operation current of $210 \mathrm{~A}$ for the worst operation case of the MICE channel [2].

The high level of stress inside the coupling coil and small mechanical disturbances which may induce heat may cause quench or permanent damage to the magnet due to large scale size and high magnetic field. Epoxy cracking and wire motion are considered to be the dominant types of mechanical

Manuscript received 1 August 2010. This work was supported by Funds of cryogenics and superconductivity technology innovation project under " $985-2$ Plan” of Harbin Institute of Technology, China and by the Office of Science, US Department of Energy under contract DE-AC02-05CH11231.

L. Wang is with Shanghai Institute of Applied Physics, Shanghai 201204, China (phone: 86-21-33932552, e-mail: wangli@sinap.ac.cn).

H. Pan, X. L. Guo, H. Wu, S. X. Zheng, are with the Institute of Cryogenics and Superconductivity Technology, Harbin Institute of Technology, Harbin 150001, China ( e-mail: panheng@hit.edu.cn).

M. A. Green is with Lawrence Berkeley National Laboratory, Berkeley, CA, 94720 USA (e-mail: magreen@lbl.gov). disturbances to cause instability of magnets impregnated using epoxy resin [3][4]. The crack of epoxy often happens in the region of stress concentration in the coil. It will cause the decrease of rigidity and the increase of deformation, and then releases the stored strain energy as heat on adjacent conductors. The large Lorentz forces during the excitation of the magnet can bring about sudden motions of wire, which will eventually release frictional energy in the coil. The MICE coupling magnet is designed to operate at liquid helium temperature. However, heat capacities of materials are so small and small thermal disturbance is possible to be a cause of quench. So, the dissipated energy resulting from strain energy or frictional energy will trigger a quench when it is larger than the minimum quench energy (MQE) of the magnet.

The coupling magnet has $13 \mathrm{MJ}$ of stored energy at $210 \mathrm{~A}$ for the worst operation case, and the hot spot temperature can be up to $130 \mathrm{~K}$ during a quench. Therefore, it is useful to study the mechanical disturbances and their effects on the magnet stability, and then optimize the magnet structure design accordingly to avoid possible instability. This paper presents the detailed quantitative analyses on epoxy cracking and conductor motion in the coupling coil assembly after the calculation of MQE, in order to study the mechanical instability of the magnet.

\section{StRuCture OF the MICE COUPLing MAgnet}

The MICE coupling coil assembly can be divided into coil package, banding, mandrel and insulations. The coil package consists of fiberglass cloth, epoxy resin, and superconductors, which is wrapped by 6061 aluminum alloy wire as banding. The inner radius of the coil is $750 \mathrm{~mm}$ and its thickness is 104 $\mathrm{mm}$ at room temperature. The mandrel made by a 6061-T6 aluminum alloy includes one bobbin, two end plates,

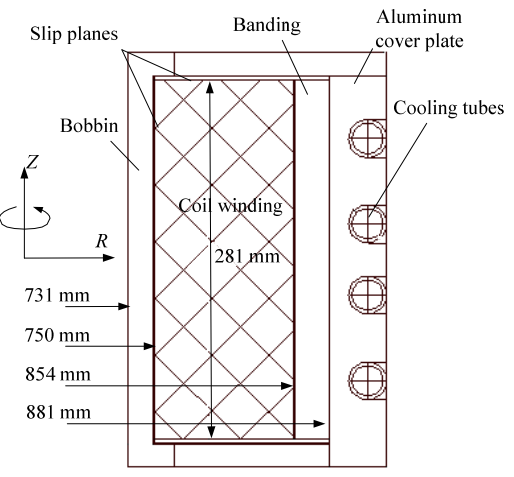

Fig. 1. The cross section of MICE coupling coil assembly 
and one cover plate in thickness of 19, 18, and $45 \mathrm{~mm}$, respectively, as well as the G-10 insulation plates between the coil and the mandrel. The cooling cryogen flows in the cover plate through nested piping to cool the coil by heat conduction. The cross section of the MICE coupling coil assembly is shown in Fig. $1 . Z$ is the axial direction, and $R$ is the radial direction.

The superconductor is wound on the aluminum bobbin under a constant tension up to $96 \mathrm{~N}(60 \mathrm{MPa})$ at room temperature. To release the shear stress and prevent conductor from moving rapidly, two layers of 50 micrometer Kapton films are to be applied as the bottom slip plane between the coil and the bottom G-10 plates. The insulations between the coil and the mandrel end plates will adopt a 0.5 mm thick G-10 sheet and a 3 mm thick G-10 plate.

\section{Minimum QUENCH ENERGY CALCULATION}

To prevent a magnet from quench, it is important to identify the MQE in the magnet. Hence, MQE is defined as the largest instantaneous energy depositing at a point of the coil from which the conductor can still recover superconducting state. That is the minimum energy to make a superconductor quench [5][6], therefore, it is the index of transient stability of a superconducting magnet, which means the stability of magnets will increase with increasing of the MQE.

MQE is related to the microcosmic structure instead of cooling method for an adiabatic stabilized magnet. Because the coil package is a kind of composite structure, it is treated as anisotropic and the effect of epoxy on the thermal conductivity of the coil in axial and radial directions is considered. The heat transfer rate in hoop direction of conductor itself is much larger than that in axial and radial directions, the estimate of MQE just considering the heat transfer in axial and radial directions will be a conservative value in design. Therefore, the coil is simplified as one 2-D axisymmetric model to simulate the transient process after a disturbance injected. The heat conduction differential equation at unsteady state for the coil is:

$C_{p} \frac{\partial T}{\partial t}=\frac{1}{r} \frac{\partial}{\partial r}\left(r \lambda_{r} \frac{\partial T}{\partial r}\right)+\frac{\partial}{\partial z}\left(\lambda_{z} \frac{\partial T}{\partial z}\right)+\beta \rho_{\text {coil }}(T) J^{2}$

In the above formula, $C_{p}$ is the coil average volumetric specific heat; $t$ is time; $\lambda_{r}$ and $\lambda_{z}$ are the effective thermal conductivities of the coil in radial and axial directions; $\beta$ is the filling factor of the coil, which is the volume ratio of the conductor and the coil package; $J$ is the current density; $\rho_{\text {coil }}$ is the average resistivity of the coil, which is the function of temperature given by:

$$
\rho_{\text {coil }}(T)=\left\{\begin{array}{cc}
0 & T<T_{\text {sh }} \\
\frac{T-T_{\text {sh }}}{T_{\mathrm{c}}-T_{\mathrm{sh}}} \rho_{\mathrm{c}} & T_{\mathrm{sh}}<T<T_{\mathrm{c}} \\
\rho_{\mathrm{c}} & T_{\mathrm{c}}<T
\end{array}\right.
$$

where $T_{\mathrm{sh}}$ is the current sharing temperature, the value is $5 \mathrm{~K} ; T_{\mathrm{c}}$ is the critical temperature of the conductor, which is $5.8 \mathrm{~K}$; $\rho_{\mathrm{c}}$ is the resistance of copper at 5.8 20 K. Here, the finite element method (FEM) was used to solve Eq. (1), and the grid of the finite element model is shown in Fig. 2. The model is adiabatic and used ANSYS as its simulation environment, and used the element of plane 55. There are about 20000 nodes in the model. The interface conductivity is managed by thermal contact elements. The multi-steps method with the Jacobi Conjugate Gradient (JCG) solver was adopted in the model.

In the FE model, the initial point of a quench is in the mid-plane of the innermost layer of the coil. The heat transfer to the cooling channel is not considered since there is adiabatic conditions and very short time of the process. The pulse width of the initial disturbance energy is $0.02 \mathrm{~s}$, which affected the the amount of the distuebance energy. The initial temperature of the coil assembly is uniform at $4.2 \mathrm{~K}$, and the thermal conductivity of the coil in axial and radial directions are about $1.6 \mathrm{~W} /(\mathrm{mK})$ and $0.3 \mathrm{~W} /(\mathrm{mK})$, respectively. The cross area of the initial point (as a heater) is about $2 \mathrm{~mm}^{2}$.

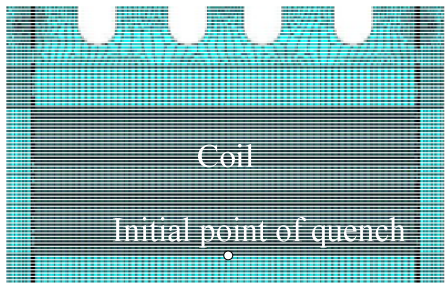

Fig. 2. Grid of the 2-D model for calculation of the MQE

At the initial time after the disturbance is applied, the critical current at the start point decreases to some value below the transient current to trigger a quench. As shown in Fig. 3, whenthe initial disturbance energy is less than $270 \mathrm{~mJ}$, the normal zone generated from the start point will reduce due to the cooling from the surroundings. When the initial disturbance energy is larger than $290 \mathrm{~mJ}$, the hot spot temperature increased following the process of decline. It indicates that the heat-generating ability of the local normal zone from hot spot is larger than the cooling capacity of surroundings as time goes on, and then the normal zone will propagate and finally the coil quenches. Therefore, the MQE of the coupling magnet can be estimated as $280 \mathrm{~mJ}$, namely, the minimum quench energy density is $3 \times 10^{4} \mathrm{~J} / \mathrm{m}^{3}$. If the disturbance energy is less than the MQE, the magnet will keep stable.

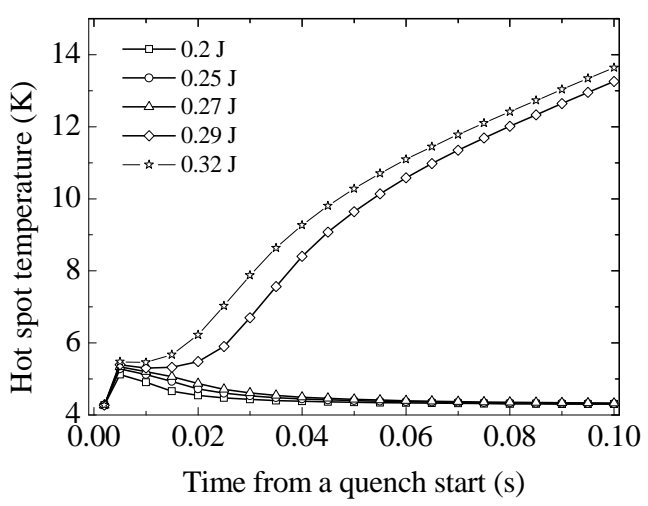

Fig. 3. Hot spot temperature vs. disturbance energy

\section{Mechanical Disturbance Calculation}

\section{A. Dissipated Strain Energy of Epoxy Cracking}

Epoxy cracking is inevitably induced due to high stress inside the coil, especially in the region of stress concentration at corners of the innermost layer. Epoxy has low fracture strength 
at cryogenic temperature. It will crack when tensile or shear stress is larger than its ultimate strength or fracture strength. The cracks result in the stored elastic energy in epoxy to dissipate as heat into the windings and the degradation of the rigidity of the superconducting windings. If the dissipated strain energy is larger than the MQE, a quench will happen.

The main failure modes of epoxy at cryogenic temperature are tensile mode and shearing mode [7]. The failure factor of the former is the major principal stress, and that of the latter is the maximum shear stress. The epoxy to be used in the coupling magnet has the tensile strength and the shear strength of 23 $\mathrm{MPa}$ and $30 \mathrm{MPa}$, respectively [8]. The fracture strength is:

$\sigma_{\mathrm{C}}=\sqrt{\frac{2 E \zeta}{\pi\left(1-v^{2}\right) a_{\mathrm{c}}}}$

where $\zeta$ is energy to failure of epoxy, which is about $860 \mathrm{~J} \cdot \mathrm{m}^{-2}$; $E$ is the elastic modulus of epoxy, which is $4 \mathrm{GPa}$; $v$ is Poisson's ratio of $0.2 ; a_{\mathrm{c}}$ is crack length of $2 \mathrm{~mm}$ according to the tensile test of epoxy. Therefore, the fracture strength of epoxy is estimated to be $34 \mathrm{MPa}$.

The main concentrated component in the region of stress concentration is shear stress. The stress status of epoxy in the region of stress concentration is investigated by principal stress method. The principal stress of epoxy in the stress concentration region can be calculated by:

$\sigma_{\mathrm{p}}^{3}-\Theta_{1} \sigma_{\mathrm{p}}^{2}+\Theta_{2} \sigma_{\mathrm{p}}-\Theta_{3}=0$

In the formula (4a), $\sigma_{\mathrm{p}}$ is a principal stress; $\Theta_{1}, \Theta_{2}$ and $\Theta_{3}$ are the $1^{\text {st }}, 2^{\text {nd }}$ and $3^{\text {rd }}$ stress invariant which is calculated by:

$\Theta_{1}=\sigma_{r}+\sigma_{\theta}+\sigma_{z}$

$\Theta_{2}=\sigma_{r} \sigma_{\theta}+\sigma_{\theta} \sigma_{z}+\sigma_{r} \sigma_{z}-\tau_{r z}^{2}$

$\Theta_{3}=\sigma_{r} \sigma_{\theta} \sigma_{z}-\sigma_{\theta} \tau_{r z}^{2}$

The maximum shear stress by the principal stresses is:

$\tau_{\max }=\frac{\sigma_{1}-\sigma_{3}}{2}$

where $\sigma_{1}$ and $\sigma_{3}$ are the major principal stress and the third principal stress, respectively.

The stresses in the region of stress concentration with and without slip planes after the coil are charged to $210 \mathrm{~A}$ are listed in the Table I [2].

TABLE I SRESSES IN THE REGION OF STRESS CONCENTRATION

\begin{tabular}{ccc}
\hline \hline Stress & With Slip Planes (MPa) & Without Slip Planes (MPa) \\
\hline$\sigma_{r}$ & -2 & -2.4 \\
$\sigma_{\theta}$ & -70 & -75 \\
$\sigma_{z}$ & -20 & -20 \\
$\tau_{r z}$ & 26 & 55 \\
\hline
\end{tabular}

In the case without slip planes, the major principal stress in the region of stress concentration is about $39 \mathrm{MPa}$, and the maximum shear stress is about $62 \mathrm{MPa}$, which are much larger than the fracture strength and the shear strength, and the failure is mainly due to shearing. In the case with slip planes, the major principal stress in the region of stress concentration is about 14 $\mathrm{MPa}$, and the maximum shear stress is about $42 \mathrm{MPa}$. That means the epoxy may also crack, but the strain energy is smaller than that without slip planes.

The strain energy density in the region of stress concentration without slip plane is [9]:

$\psi_{\mathrm{C}}=\frac{3 \sigma_{c}^{2}(1-2 v)}{2 E}$

The strain energy density is about $7.6 \times 10^{4} \mathrm{~J} / \mathrm{m}^{3}$ and 50 percent of the strain energy is assumed to dissipate into the adjacent conductors, which is larger than the minimum quench energy density of the coupling coil. Therefore, quench or training behavior will be induced at the corners of the coil innermost layer due to epoxy crack caused by shear stress.

\section{B. Dissipated Frictional Energy of Conductor Motion}

It is generally understood that the sudden conductor motion will induce heat. In an impregnated coil, the impregnate such as epoxy is filled into the inter space of turns, and the conductors are supported by the frictional force between impregnated material and conductors. The frictional force is enhanced by means of winding pre-tension to be applied on the conductors during winding.

For the coupling magnet, because of large scale size, there may be incomplete filling of epoxy between turns especially at the ends of layers, and then the frictional force in the defective sections decreases. When the magnet is charged, the conductors in the defective sections will bend along the axial direction due to the axial Lorentz force, and if the frictional force is less than the local Lorentz force, the conductor motion will happen. The abrupt moving usually only lasts a few microseconds. The dissipated frictional energy can not be carried away by refrigeration immediately, and quench will be induced when the frictional energy is larger than the MQE.

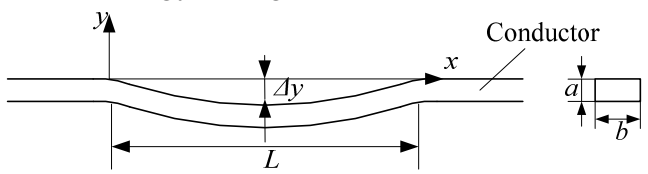

Fig. 4. Illustration of the conductor motion in the coil winding

The illustration of conductor bending and moving due to incomplete filling of epoxy or gap between turns is shown in Fig. 4. If the length of the defective section between conductors is $L$, and the bent conductor is treated as a beam, according to the theory of bending beam, the displacement of conductor is:

$y(x)=\frac{B_{z} I x^{2}(x-L)^{2}}{24 E_{\mathrm{c}} I_{\mathrm{cd}}}$

where $B_{z}$ is the axial magnetic induction; $I$ is the operation current; $E_{\mathrm{c}}$ is the elastic modulus of conductor; $I_{\mathrm{cd}}$ is the geometrical moment of inertia. For a rectangular section conductor, it is:

$I_{\mathrm{cd}}=\frac{a b^{3}}{12}$

The axial Lorentz force makes the conductor move $y(x)$, part of the work is stored in the conductor in the form of strain energy, and the other is to overcome the frictional force and dissipate frictional energy to the adjacent conductors.:

$\Pi_{\mathrm{cm}}(L)=f_{\mathrm{cf}} \cdot \int_{0}^{L} y(x) \mathrm{d} x$

where $f_{\text {cf }}$ is the density of local frictional force on the bending 
conductor. If the Lorentz force is entirely used to overcome the frictional force, the relationship of the bending length and frictional energy at different locations of the coil is shown in Fig. 5.

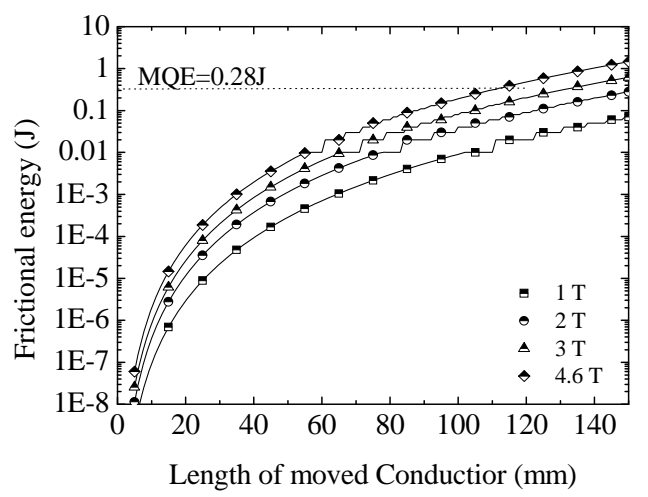

Fig. 5. Relationship of the bending length and frictional energy at different magnetic fields

The maximum axial magnetic induction locates at the ends of the coil. Thus, the axial Lorentz force at both ends of the coil is the largest. As shown in Fig. 5, in order to limit the dissipated frictional energy due to conductor motion less than the MQE, the defective length between conductors should be less than $100 \mathrm{~mm}$ at both ends of the coil. However, during the actual process of winding, the incomplete filling easily occurs at the transition location from one layer to the next layer. Therefore, the defective length between conductors is an important factor controlling the index in winding process and it is required that the defective length should be less than $100 \mathrm{~mm}$ for the conductors and the requirement can be reduced for the conductors in the middle of the coil due to a relative smaller axial Lorentz force.

\section{EFFect of Slip Planes on Mechanical Stability}

The slip planes between the coupling coil and the mandrel mainly have a great effect on the transverse shear stresses induced by the thermal contraction during cool down process [10]. According to the analyses, the slip planes can remarkably reduce the cracking range of epoxy and the dissipated energy.

The conductors on the slip plane will also move when the axial Lorentz force is larger than the frictional force between the innermost conductors and the slip plane. Unlike the conductors in the outer layers, the innermost layer will slow slip relatively to the bobbin during charging, and the frictional energy will dissipate into each innermost conductor. That means each innermost conductor can be treated as a single heat source. If the dissipated frictional energy in each conductor is less than the MQE, the "slow-slip" will not induce quench.

The relative displacement between the innermost conductor and the bobbin is shown in Fig. 6. The maximum relative displacement is about $16 \mu \mathrm{m}$ which happens at the both ends of the coil. For any given axial position $z_{0}$, the frictional energy density on the slip plane is:

$E_{\mathrm{f}}\left(z_{0}\right)=\frac{2 \mu P\left(z_{0}\right) \delta\left(z_{0}\right)}{V_{\mathrm{c}}} A_{\mathrm{c}}$

where $\mu$ is the friction coefficient of slip plane; $P\left(z_{0}\right)$ is the contact stress of slip plane; $\delta\left(z_{0}\right)$ is the relative displacement; $A_{\mathrm{c}}$ and $V_{\mathrm{c}}$ are the friction area and conductor volume.

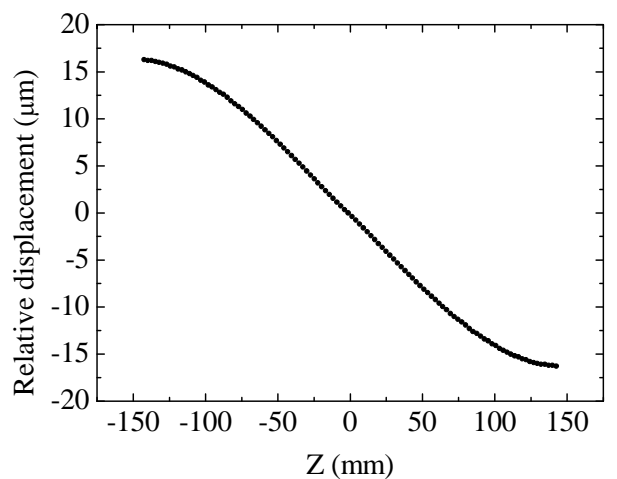

Fig. 6. Relative displacement between the innermost conductor and the bobbin

The maximum frictional energy in the innermost conductors is about $7230 \mathrm{~J} / \mathrm{m}^{3}$, which is less than the MQE. That means the dissipated energy in each conductor can be carried away by cooling from the surroundings. The slow-slip on the slip plane will not induce quench.

\section{CONCLUSION}

The quantitative analyses on epoxy cracking and conductor motion are discussed in this paper. The MQE of the coupling magnet is calculated by a finite element model. The cracking of epoxy is the main mechanical disturbance for the coupling magnet. Slip planes have a great effect on the reduction of the epoxy cracking range and strain energy, which may reduce the possibility of quench resulting from crack of epoxy resin inside the coil assembly. It is found that the defective length between conductors should be limited within $100 \mathrm{~mm}$ to avoid quench. According to the calculations, the slow-slip on the slip plane during coil charging will not induce quench.

\section{REFERENCES}

[1] G. Gregoire, G. Ryckewaert, and L. Chevalier et al., "MICE and International Muon Ionization Cooling Experiment Technical Reference Document,” [Online]. Available: http://hep04.phys.iit.edu/cooldemo.

[2] Institute of cryogenics and superconductivity Technology, "Muon Ionization Cooling Experiment Coupling Solenoid Magnet Engineering Design Report,” (Unpublished) Harbin Institute of Technology, China, Dec. 2008.

[3] H. Miyamoto, S. Nisliijima, and T.Kushida et al. "Instability of impregnated superconducting windings induced by mechanical disturbance,” IEEE Trans. Magn., vol. 32, pp. 3008-3011, July 1996.

[4] Y. Iwasa, "Mechanical disturbances in superconducting magnets-a review," IEEE Trans. Magn. vol. 28, pp. 113-120, Jan. 1992.

[5] L. Dresner. "Quench energies of potted magnets," IEEE Trans. Magn. vol. MAG-21, pp. 392-395, Aug. 1985.

[6] K. Seo; M. Morita; and S. Nakamura et al, "Minimum quench energy measurement for superconducting wires,” IEEE Trans. Magn. vol. 32, pp. 3089 -3093, Jul. 1996.

[7] Z. Fu, Strength and Damage Behavior of High Polymer Material, Beijing: Chemical Industry Press, 2005, pp. 52-67.

[8] S. Yadav, J. Hoffman, Epoxies for Cryogenic Applications, Fermi Lab Note, TD-99-021, 1999.

[9] M. N. Wilson. Monographs on Cryogenics Superconducting Magnets. Oxford: Oxford University Press, 1983, pp. 85-89.

[10] L. Wang, H. Pan, H. Wu et al. "Effects of Slip Planes on Stresses in MICE Coupling Solenoid Coil Assembly,” IEEE Trans. Supe. vol. 20, pp. 1940 -1943 , Jun. 2010. 


\section{DISCLAIMER}

This document was prepared as an account of work sponsored by the United States Government. While this document is believed to contain correct information, neither the United States Government nor any agency thereof, nor the Regents of the University of California, nor any of their employees, makes any warranty, express or implied, or assumes any legal responsibility for the accuracy, completeness, or usefulness of any information, apparatus, product, or process disclosed, or represents that its use would not infringe privately owned rights. Reference herein to any specific commercial product, process, or service by its trade name, trademark, manufacturer, or otherwise, does not necessarily constitute or imply its endorsement, recommendation, or favoring by the United States Government or any agency thereof, or the Regents of the University of California. The views and opinions of authors expressed herein do not necessarily state or reflect those of the United States Government or any agency thereof or the Regents of the University of California. 